\title{
Asociación entre el estado nutricional y el retardo eruptivo en niños de 6 a 12 años. Piura Perú 2018
}

Association between nutritional status and delay in the eruption in children from 6 to 12 years old. Piura Perú 2018

Milton Cea-Sanhueza ${ }^{1, a}$, Bastian Godinez-Pacheco ${ }^{1, a}$, Carlos Araya-Vallespir ${ }^{2, b}$, César Del-Castillo-López ${ }^{3, c}$.

\section{RESUMEN}

El estado nutricional (EN) del niño/a es importante dado el aporte de nutrientes esenciales en el desarrollo de estructuras craneofaciales, que al disminuir comprometen a todo el organismo. Una manera de monitorear y prevenir los efectos de la malnutrición es mediante la valoración antropométrica talla para la edad (T/E) e IMC para la edad (IMC/E). Objetivo: Determinar la asociación el EN y el retardo eruptivo (RE) de piezas permanentes y EN en niñas y niños entre 6 a 12 años de los distritos de Canchaque y San Miguel de El Faique en los meses de junio y julio de 2018. Material y métodos: 354 niños de 6 a 12 años de los distritos de Canchaque y San Miguel del Faique fueron evaluados el año 2018 y se registraron las variables sociodemográficas, EN y RE. Se analizan las prevalencias y se asocian. Se reportan las variables en tablas de frecuencias y se asocian EN con RE por medio de tablas de contingencia y calculando el OR y RP. Resultados: La prevalencia de individuos con al menos una variable antropométrica (VAM) deficiente es $43 \%$ y RE presente es $26 \%$. Se encontró una asociación significativa (O.R. $=2,42 \mathrm{IC}=1,49-3,91)(\mathrm{RP}=1.90 \mathrm{p}<0.001)$ entre la VAM T/E y RE. Conclusiones: La prevalencia de casos con RE fue mayor a la de otros estudios. Hay asociación entre el EN de los individuos y el RE, especialmente la VAM T/E y el RE.

PALABRAS CLAVE: Desnutrición, erupción dental, salud bucal, Perú.

\section{SUMMARY}

The nutritional status (NS) of a child is important since there are essential nutrients needed for the development of craniofacial structures that, when low, compromise the body. One way to monitor and prevent the effects of malnutrition is with the age-appropriate anthropometric assessment (height-for-age) and BMI-for-age. Objective: Determine the association between delayed eruption (DE) of permanent teeth and NS in children aged 6 to 12 years from the districts of Canchaque and San Miguel de El Faique at the months of June and July 2018. Material and methods: 354 children aged 6 to 12 years old from the districts of Canchaque and San Miguel de El Faique are examined and the sociodemographic variables, NS and DE are recorded. Prevalences are analyzed and assessed for associations. Variables are reported in frequency tables and associated NS with DE through contingency tables and calculating their OR And RP. Results: The prevalence of individuals with at least a deficient anthropometric valuation (VAM) is $43 \%$, and $\mathrm{DE}$ is $=26 \%$. When assessing relationships, a statistically significant association $(\mathrm{O} . \mathrm{R} .=2.42 \mathrm{IC}=1.49-3.91)(\mathrm{RP}=1.90 \mathrm{p}<0.001)$ between the VAM-heightfor-age and DE is described. Conclusions: The prevalence of DE cases was higher than in other studies. There is an association between the NS of the individuals and DE with a positive statistical correlation between them, especially a VAM height-for-age and DE.

KEY WORD: Malnutrition, tooth eruption, oral health, Perú.

\footnotetext{
${ }^{1}$ Facultad de Odontología, Universidad de Concepción. Concepción, Chile

2 Dpto. Prevención y Salud Pública Odontológica, Facultad de Odontología, Universidad de Concepción. Concepción, Chile

${ }^{3}$ Departamento Académico de Odontología Social, Facultad de Estomatología "Roberto Beltrán", Universidad Peruana Cayetano Heredia. Lima, Perú.

a Estudiante pregrado

${ }^{\mathrm{b}}$ Dr. Profesor Asociado, Director

${ }^{c} \mathrm{Mg}$. Docente
} 


\section{INTRODUCCIÓN}

El estado nutricional (EN) de un individuo es considerado una de las condiciones más importantes durante la infancia considerando su influencia en el desarrollo físico, bioquímico y mental (1). Es por ello que la correcta alimentación es importante y más aún el oportuno diagnóstico de las alteraciones nutricionales que constituyen un riesgo para la salud general y bucal (2-4). Así lo demuestran reportes que relacionan el EN con otros indicadores biológicos de la salud afectándose mutuamente y perpetuando una disminución en la calidad de vida del individuo (4).

Algunos autores sugieren que el descuido de atención en salud durante el embarazo y parto significan un factor de riesgo importante en la desnutrición de niños recién nacidos (5). Sin embargo, ha de entenderse que en la realidad latinoamericana la malnutrición es una de las principales consecuencias de la desigualdad socioeconómica, falta de educación y pobre acceso a una alimentación saludable (2).

La existencia de una relación entre individuos con al menos un indicador de enfermedad en el estado de salud bucal y el EN se ha observado en individuos adultos y niños, donde un EN deficiente durante el desarrollo afecta de manera directa al estado de salud bucal (7-9). Esto debido al déficit de nutrientes, esenciales para el desarrollo craneofacial, como son el ácido fólico (10), calcio, fluoruros y vitaminas del complejo B, A, C y especialmente el D, que juega un rol importante en el desarrollo, calcificación y mantenimiento de estructuras (11). Por otra parte, se ha descrito cómo una dieta balanceada y adecuada se convierte en un factor protector de la salud periodontal(11).

La secuencia eruptiva de piezas dentarias ha sido blanco de estudio por diversos autores, sin embargo, es difícil hablar de cifras definitivas en tanto estos estudios han demostrado que dicha secuencia y tiempo es influenciada por el sexo, genética, condición socioeconómica y nutricional.(12) Sin embargo, en lo dicho a factor nutricional, son pocos los estudios que se han realizado consiguiendo demostrar que el retardo eruptivo (RE) de dientes permanentes (como evento propio del desarrollo y crecimiento del niño) se ve asociado a alguna alteración en sus mediciones antropométricas específicas y la malnutrición por déficit (13) (14).

Un organismo mal nutrido es sinónimo de un sistema inmune deficiente (15) que frente a un proceso infeccioso (como la enfermedad periodontal o el curso de una caries sin tratar) se presta a una respuesta inadecuada (16). Por tanto, hay una posibilidad de observar estudios que relacionan dolencias bucales y desnutrición existiendo asociación o no entre ellas $(1,15-17)$.

La malnutrición por déficit (MPD), es un estado pluricarencial donde predomina el déficit protéico energético y no se provee al organismo de suficientes metabolitos que cubran las necesidades para el funcionamiento, crecimiento y desarrollo normales (17); y para la cual la UNICEF promueve métodos de examen como son las valoraciones antropométricas (VAM) peso para la edad, peso para la talla, talla para la edad (T/E) e IMC para la edad (IMC/E) (18).

En Perú la prevalencia de MPD en la infancia ha mostrado una caída de casi 10 puntos desde el año 2005 $(29,8 \%)$ al $2011(18,1 \%$.) gracias a los esfuerzos que programas sociales como las transferencias de efectivo condicional (JUNTOS), la plataforma de trabajo de la sociedad civil que priorizan las intervenciones nutricionales (CRECER) o la promoción de la lactancia materna; que han llevado a la población a entender la importancia de una correcta alimentación (19).

Una consecuencia de la MPD es el retraso del desarrollo, situación clínica en que el niño deja de progresar en comparación al ritmo esperado para su edad (20). A fin de prevenir aquello existe el monitoreo del estado nutricional mediante mediciones antropométricas como peso y talla, contrastadas con la edad e Índice de Masa Corporal (IMC). Así se obtiene una VAM que puede ser: Peso para la edad, Peso para la talla y T/E que refleja el crecimiento lineal alcanzado en relación con la edad cronológica. E IMC para la edad IMC/E que refleja el peso relativo con la talla para cada edad. Las mencionadas son VAM indicadas por la OMS para la evaluación nutricional de niños menores a 6 años (18), mientras que para mayores, se encuentran indicadas las VAM T/E e IMC/E.

El objetivo de este trabajo fue determinar la asociación entre el retardo eruptivo de piezas permanentes y estado nutricional en niñas y niños entre 6 a 12 años de los distritos de Canchaque y San Miguel de El Faique a los meses de junio y julio de 2018. 


\section{MATERIAL Y MÉTODOS}

Se diseñó y realizó un estudio de tipo transversal aplicado a una muestra no aleatoria constituida por la recolección de datos permitida durante un periodo de tiempo de 2 meses resultando en 354 niños en etapa escolar de 6 a 12 años de edad pertenecientes a los centros poblados de los distritos de Canchaque y San Miguel de El Faique al año 2018. Los criterios de inclusión fueron; vivir en el distrito de Canchaque o San Miguel de El Faique, cursar estudios en el centro poblado capital del distrito o caseríos aledaños, contar con la autorización y consentimiento informado del tutor para la participación y estar presente en la jornada de examinación. Mientras que el criterio de exclusión fue poseer alguna condición sindrómica diagnosticada que afecte a su talla, peso o estado de salud bucal.

Se solicitaron los permisos y autorizaciones correspondientes, junto con la presentación del protocolo de investigación al comité de bioética institucional de la Universidad de Concepción, año 2018. Debido a que la mayoría de los autores cuentan con la filiación de la Universidad de Concepción. Se efectuaron consentimiento informado a los padres y apoderados de los niños a examinar.

Para determinar el EN del individuo se recopilaron indicadores como sexo, edad, peso y talla; estos últimos con el uso de una balanza digital la que fue verificada previo uso y una cinta métrica metálica adherida a una pared de forma perpendicular al piso como sugiere la UNICEF (18). La valoración posterior de estas variables fue realizada utilizando las tablas de VAM de varones y mujeres entre 5 y 17 años del Ministerio de Salud del Perú, registrando tanto la VAM $\mathrm{T} / \mathrm{E}$ e IMC/E por cada individuo. Al momento de tabular esta variable se agruparon aquellos individuos que presentan riesgo de déficit, déficit y déficit severo de T/E e IMC/E en un grupo denominado "normal - baja" de forma independiente para cada VAM. Y aquellos que presentan normalidad, riesgo de exceso, exceso y exceso severo en un grupo denominado "normal - alta" para cada VAM respectivamente.

Para determinar los RE de piezas permanentes se registraron en la ficha de examen aquellas piezas no erupcionadas y las que al momento del examen se encontraban en proceso de evidente erupción pre-funcional (EPF) (hasta media corona clínica sin contacto con el antagonista). De este modo, posteriormente se pudo contabilizar el número de RE por individuo identificando aquellas piezas no erupcionadas que ya deberían encontrarse al menos en proceso de EPF, utilizando como referencia la tabla de erupción dental de la $\operatorname{ADA}(21)$, y considerando aquellas que se encontraban en proceso de EPF al momento del examen como si cuya aparición en boca se hubiese dado en un plazo anterior de seis meses, basados en la duración del proceso de EPF más largo (1 año) registrado para la pieza más voluminosa en boca (primer molar) (22), lo que permite saber si al momento de su erupción en la encía se encontraba fuera del plazo. Adicionalmente se contabilizan y registran el número de piezas retardadas por individuo.

Para efectuar el examen bucal a los individuos se utilizó espejo bucal número 5 con iluminación frontal y en condiciones de luz ambiental favorable, siguiendo los criterios de examen propuestos por la OMS en su documento Oral Health Surveys - Basic Methods $5^{\text {ta }}$ edición(23).

Los examinadores fueron calibrados mediante una sesión de discusión de criterios y práctica en el que debieron alcanzar un nivel de concordancia al menos $\mathrm{p}=0,8$.

Los datos fueron tabulados en Excel y exportados al paquete estadístico Infostat para su posterior análisis. Se comenzó con una descripción de variables sociodemográficas, RE y EN con tablas de frecuencia. Para explorar asociaciones se utilizó tablas de contingencia entre EN con RE. Para cuantificar la asociación se obtuvo el OR de T/E con RE e IMC/E con RE y sus respectivos intervalos de confianza.

\section{RESULTADOS}

Del grupo de individuos examinados la tabla 1 y 2 muestran las frecuencias de variables sociodemográficas procedencia, sexo y edad en la población estudiada. Se puede apreciar una mayor participación de individuos del distrito de Canchaque y una similar distribución de sexos. Adicionalmente, la edad media de la población es de 9 años $\pm 1,9$ años (tabla 1 ).

En relación a la prevalencia de las variables RE y EN, la tabla 2 permite visualizar la presencia o no de RE y la cantidad de VAM (T/E y/o IMC/E) que se encuentran deficientes; en la población total, segregado según sexo y edad. Información que se complementa con la tabla 3 que muestra prevalencia de VAM para la población total y según sexo. De este modo, se 
Tabla 1. Frecuencia de las variables sociodemográficas de la población

\begin{tabular}{|c|c|c|}
\hline Distrito & & n $(\%)$ \\
\hline Canchaque & & $205(57,9)$ \\
\hline San Miguel de El Faiqu & & $149(42,1)$ \\
\hline & Total & $354(100,0)$ \\
\hline Sexo & & n $(\%)$ \\
\hline Femenino & & $181(51,1)$ \\
\hline \multirow[t]{2}{*}{ Masculino } & & $173(48.9)$ \\
\hline & Total & $354(100,0)$ \\
\hline Edad (años) & & n $(\%)$ \\
\hline 6 & & $48(13,6)$ \\
\hline 7 & & $44(12,4)$ \\
\hline 8 & & $48(13,6)$ \\
\hline 9 & & $68(19,2)$ \\
\hline 10 & & $62(17,5)$ \\
\hline 11 & & $41(11,6)$ \\
\hline \multirow[t]{2}{*}{12} & & $43(12,1)$ \\
\hline & Total & $354(100,0)$ \\
\hline
\end{tabular}

Tabla 2. Frecuencia de estado nutricional y retardo en la erupción para la población total según sexo y edad

\begin{tabular}{|c|c|c|c|c|c|c|c|c|c|c|}
\hline & \multirow[t]{2}{*}{ Total } & \multicolumn{2}{|c|}{ Sexo } & \multicolumn{7}{|c|}{ Edad } \\
\hline & & $\mathbf{F}$ & M & 6 & 7 & 8 & 9 & 10 & 11 & 12 \\
\hline $\begin{array}{l}\text { Estado } \\
\text { nutricional } \\
\text { (número de } \\
\text { valoraciones } \\
\text { deficientes) }\end{array}$ & n (\%) & n (\%) & n (\%) & n (\%) & n (\%) & n (\%) & n (\%) & n (\%) & n (\%) & n (\%) \\
\hline 0 & $201(57)$ & $99(55)$ & $102(59)$ & $30(63)$ & $23(52)$ & $32(67)$ & $37(54)$ & $33(53)$ & $22(54)$ & $24(56)$ \\
\hline 1 & $130(37)$ & $69(38)$ & $61(35)$ & $15(31)$ & $16(36)$ & $15(31)$ & $27(40)$ & $26(42)$ & $16(39)$ & $15(35)$ \\
\hline \multirow[t]{3}{*}{2} & $23(6)$ & $13(7)$ & $10(6)$ & $3(6)$ & $5(11)$ & $1(2)$ & $4(6)$ & $3(5)$ & $3(7)$ & $4(9)$ \\
\hline & Total & \multicolumn{2}{|c|}{ Sexo } & & & & Edad & & & \\
\hline & & $\mathbf{F}$ & $\mathbf{M}$ & 6 & 7 & 8 & 9 & 10 & 11 & 12 \\
\hline $\begin{array}{l}\text { Retardo } \\
\text { eruptivo }\end{array}$ & n (\%) & n (\%) & n (\%) & n (\%) & n (\%) & n (\%) & n (\%) & n (\%) & n (\%) & n (\%) \\
\hline $\mathrm{Si}$ & $92(26)$ & $28(15)$ & $64(37)$ & $0(0)$ & $10(23)$ & $9(19)$ & $11(16)$ & $33(53)$ & $16(39)$ & $13(30)$ \\
\hline No & $262(74)$ & $153(85)$ & $109(63)$ & $48(100)$ & $34(77)$ & $39(81)$ & $57(84)$ & $29(47)$ & $25(61)$ & $30(70)$ \\
\hline
\end{tabular}


puede ver que la mayor parte de la población (74\%) no presenta RE y se encuentra en un EN sin ninguna VAM deficiente (57\%).

La VAM deficiente que mayor frecuencia poblacional presenta es T/E con un 40\% (tabla 3), contra la VAM deficiente IMC/E que representa solo el 10\%. De esta manera, la variable EN (tabla 4) muestra que el 43\% de los individuos examinados presenta al menos una de ambas VAM deficiente.

Al estudiar de forma separada el riesgo de RE según VAM T/E e IMC/E se obtiene un $\mathrm{OR}=2,4$; IC $95 \%=[1,5-3,9]$ y OR $=1,6$; IC $95 \%=[0,8-$ $3,2]$ respectivamente. Utilizando RP se obtuvo 1.90 $(\mathrm{p}<0.001)$ y $1.37(\mathrm{p}=0.238)$ respectivamente.

Tabla 3. Frecuencia valoración nutricional total según sexo

\begin{tabular}{ccccc}
\hline \multirow{2}{*}{ Sub-variable } & Caregoría & Total & \multicolumn{2}{c}{ Sexo } \\
\cline { 5 - 5 } & & $\mathbf{n}(\%)$ & $\mathbf{n}(\%)$ & $\mathbf{n}(\%)$ \\
\hline \multirow{2}{*}{ VAM IMC/E } & Normal - Baja & $35(10)$ & $19(10)$ & $16(9)$ \\
\cline { 2 - 4 } & Normal - Alta & $319(90)$ & $162(90)$ & $157(91)$ \\
\hline \multirow{2}{*}{ VAM Talla/E } & Normal - Baja & $140(40)$ & $75(41)$ & $65(38)$ \\
\cline { 2 - 4 } & Normal - Alta & $214(60)$ & $106(59)$ & $108(62)$ \\
\hline
\end{tabular}

Tabla 4. Asociación retardo eruptivo y valoración $\mathrm{T} / \mathrm{E}$ e IMC/E

\begin{tabular}{|c|c|c|c|}
\hline & \multicolumn{2}{|c|}{ Valoración T/E } & \multirow{2}{*}{ Total } \\
\hline & Normal - Baja & Normal - Alta & \\
\hline $\begin{array}{l}\text { Retardo } \\
\text { Eruptivo }\end{array}$ & n $(\%)$ & n $(\%)$ & n $(\%)$ \\
\hline $\mathrm{Si}$ & $51(36,4)$ & $41(19,2)$ & $92(25,9)$ \\
\hline No & $89(63,5)$ & $173(80,8)$ & $262(74,0)$ \\
\hline \multirow[t]{3}{*}{ Total } & $140(100,0)$ & $214(100,0)$ & $354(100,0)$ \\
\hline & \multicolumn{2}{|c|}{ Valoración IMC/ } & Total \\
\hline & Normal - Baja & Normal - Alta & \\
\hline $\begin{array}{l}\text { Retardo } \\
\text { Eruptivo }\end{array}$ & n (\%) & n (\%) & n (\%) \\
\hline $\mathrm{Si}$ & $12(34,3)$ & $80(25,1)$ & $92(25,9)$ \\
\hline No & $23(65,7)$ & $239(74,9)$ & $262(74,0)$ \\
\hline Total & $35(100,0)$ & $319(100,0)$ & $354(100,0)$ \\
\hline
\end{tabular}




\section{DISCUSIÓN}

El presente estudio epidemiológico fue realizado en escolares de 6 a 12 años en los centros poblados y caseríos aledaños de dos distritos del departamento de Piura (Canchaque y San Miguel de El Faique) entre junio y julio de 2018; permite estimar la prevalencia de retardo en la erupción dental y la asociación de esta al estado nutricional de los individuos.

La prevalencia encontrada de casos con RE (26\%) fue levemente mayor a la encontrada en el estudio de Díaz et al., en que reportan secuencias eruptiva alteradas en un $21,6 \%$ de la muestra (24). También presenta una prevalencia de retardos eruptivos similar a la reportada por el estudio de Mora et al., el año 2009 en un estudio realizado con niños cubanos en que alcanza un valor cercano al $24 \%$ (25).

La prevalencia de EN deficiente en este estudio reporta un $37 \%$ de individuos con solo 1 VAM deficiente y un $6 \%$ de individuos con ambas VAM deficientes; en la que los pacientes con riesgo de delgadez, delgadez y delgadez severa agrupados representan el $10 \%$ de la población y los casos con riesgo de talla baja, talla baja y talla baja severa agrupados representan un $40 \%$ de la población.

La asociación entre el EN de los individuos y el RE desglosada por cada VAM resulta de la siguiente manera: VAM T/E y el RE $(\mathrm{OR}=2,4$; IC 95\% $=[1,5-3,9])$ evidencia que la ventaja de presentar retardo es 2,4 veces mayor en individuos que tienen T/E deficiente a aquellos que no, lo que constituye una asociación estadísticamente significativa. Contrariamente al evaluar dicha asociación según VAM IMC/E $(\mathrm{OR}=1.6$; IC $95 \%=[0,8-3,2])$ se puede observar que el riesgo es menor y con un intervalo de confianza que lo hace no concluyente. Del mismo modo es Clotilde et al., quienes reportan que el estado nutricional deficiente del niño se ve asociado a alteraciones eruptivas (25). Por otro lado, el trabajo realizado por Gabriela Díaz, et al.(24) no reporta esta asociación, tal vez, debido a que el diseño metodológico de su estudio solo valora nutricionalmente a los individuos por medio de la VAM IMC/E; lo que en nuestro estudio si coincide con sus hallazgos demostrando que la asociación de estas variables se da solo en un grado muy leve.

Una forma de entender por qué la VAM T/E presenta un riesgo tan importante para el RE es recordando que esta valoración se remite a evaluar el estado de crecimiento lineal alcanzado en relación a la edad y que su déficit se relaciona a alteraciones de salud a largo plazo, por ello, es capaz de detectar a aquellos individuos que no se están desarrollando de manera adecuada para su edad lo que es consecuencia de una alteración nutricional en edades tempranas y que acaba traduciéndose en un retraso del desarrollo del niño y de su proceso de erupción dentaria $(23,29)$.

Cabe mencionar que la importancia de considerar esta tendencia en esta población específica se asocia a la pérdida prematura de piezas temporales lo que constituye un riesgo de agudizar cuadros de malnutrición debido a la merma en funcionalidad dentaria y el empeoramiento de cuadros de mal posición dentaria en el sector posterior.

Se sugiere estructurar y aplicar un modelo de estudio epidemiológico más adecuado que asocie los retardos eruptivos de un mismo grupo etario con su historial nutricional durante los primeros años de vida y así obtener una estimación de riesgo asociado a las variables. Por otro lado, se sugiere realizar estudios nacionales que permitan determinar secuencias eruptivas propias de la región.

En conclusión, se determinó que la presencia de retardos eruptivos se ve afectada por el estado nutricional. Siendo esta relación más estrecha entre la valoración antropométrica T/E y retardos eruptivos con una probabilidad mayor de presentar retardos eruptivos cuando se obtiene una valoración antropométrica de T/E deficiente.

\section{Correspondencia}

Milton Cea-Sanhueza

Correo electrónico: miltoncea@udec.cl

\section{REFERENCIAS BIBLIOGRAFICAS}

1. Torres-Trujillo LE, Duque-Cano JA, Granada-García J, Serna-Valencia M, García-Muñoz RA. Anomalías dentales y su relación con la malnutrición en la primera infancia: un análisis crítico de literatura. Rev Nac Odontol. 2015;11(20):0-0. Doi: https://doi. org/10.16925/od.v11i20.941

2. Morinigo G, Sánchez S, Sispanov V, et al. Perfil nutricional por antropometría de niños/as menores de 5 años del sistema público de salud, 2013. Pediatría (Asunción). 2015;42(3):187-91. 
3. Zaror S, Sapunar Z, Muñoz N, Gonzáles C. Asociación entre malnutrición por exceso con caries temprana de la infancia. Rev Chil pediatría. 2014;85(4):455-61.

4. Galvez A, Rosa A, García E, et al. Estado nutricional y calidad de vida relacionada con la salud en escolares del sureste español. Nutrición Hospitalaria. 2015; 31: 734-743.

5. Hamel C, Enne J, Omer K, et al. Childhood malnutrition is associated with maternal care during pregnancy and childbirth: A Cross-Sectional Study in Bauchi and Cross River States, Nigeria. J Public health Res. 2015;4(1):408. doi: 10.4081/jphr.2015.408

6. Wu LL, Cheung KY, Lam PYP, Gao X. Oral health indicators for risk of malnutrition in elders. J Nutr Health Aging. 2018;22(2):254-61. DOI: 10.1007/ s12603-017-0887-2

7. Masterson EE, Fitzpatrick AL, Enquobahrie DA, Mancl LA, Conde E, Hujoel PP. Malnutritionrelated early childhood exposures and enamel defects in the permanent dentition: A longitudinal study from the Bolivian Amazon. Am J Phys Anthropol. 2017;164(2):416-23. DOI: 10.1002/ajpa.23283

8. Pflipsen M, Zenchenko Y. Nutrition for oral health and oral manifestations of poor nutrition and unhealthy habits. Gen Dent. 2017;65(6):36-43.

9. Paulos A, Pino P, Cavada G, Lagos C, Broussain V, Hasbún A. Fisuras labio-palatinas y fortificación de la harina con ácido fólico en Chile: An exploratory study. Rev Med Chil. 2016;144(8):1012-9.

10. Schroth RJ, Rabbani R, Loewen G, Moffatt ME. Vitamin D and dental caries in children. J Dent Res. 2016;95(2):173-9.

11. Najeeb S, Zafar MS, Khurshid Z, Zohaib S, Almas K. The Role of Nutrition in Periodontal Health: An Update. Nutrients. 2016;8(9):0-0. DOI: 10.3390/ nu8090530

12. Baragua M. Factores de riesgo de alteraciones cronológicas de la erupción dentaria en la población del municipio Baraguá. Mediciego. 2013;19(2):1-11.

13. Ayala Pérez Y, Carralero Zaldívar L de la $C$, Leyva Ayala B del R. La erupción dentaria y sus factores influyentes. Correo Científico Médico. 2018;22(4):681-94.

14. Dimaisip-Nabuab J, Duijster D, Benzian H, et al. Nutritional status, dental caries and tooth eruption in children: a longitudinal study in Cambodia, Indonesia and Lao PDR. BMC Pediatr. 2018;18(1):1-11.
15. Rytter MJH, Kolte L, Briend A, Friis H, Christensen VB. The Immune System in Children with Malnutrition-A Systematic Review. PLoS One. 2014;9(8):e105017.

16. Słotwińska SM, Słotwiński R. Host response, malnutrition and oral diseases. Part 1. Cent J Immunol. 2014;39(4):518-21.

17. Bayeux AC, Rodríguez NR, Fuentes ZC, Navarro ALQ, Leyva EC. Factores de Riesgo de malnutrición por defecto en niños de 1 a 5 años. Rev Inf Científica. 2015;91(3):433-41.

18. UNICEF. Evaluación del crecimiento de niños y niñas. Material de apoyo para equipos de atención primaria de la salud. Argentina: Gobierno de la provincia de Salta; 2012. p.86.

19. Mejía A, Haddad L. The politics of success in the fight against malnutrition in Perú. Food Policy. 2014;44:26-35.

20. Martínez C, Pedrón C. Valoración del estado nutricional. Protoc diagnóstico-terapéuticos. 2012;1:314-8.

21. ADA Division of Communications, Journal of the American Dental Association, ADA Council on Scientific Affairs. Tooth eruption: the permanent teeth. J Am Dent Assoc. 2006;137(1):127.

22. Sáenz LP, Sánchez L, Luengas MI. Proceso de erupción de los primeros molares permanentes. Revista Cubana de Estomatología. 2017;54:14-23.

23. World Health Organization Oral Health Surveys Basic Methods. 5th edition. Paris, France: WHO; 2013. p. 137.

24. Dayanna-Orahulio G, León-Manco RA. Estado nutricional y secuencia de erupción dentaria en niños menores de 12 años de edad - Aldea Infantil SOS Pachacámac - Lima, Perú. Rev Estomatológica Hered. 2014;24(4):213-9.

25. Mora C, López R, Apolinaire J. Brote dentario y estado nutricional en niños de 5 a 13 años. Medisur. 2009;7(1):1-7.

Recibido: 16-08-2019

Aceptado: 02-12-2019 